\title{
EXPERIMENTAL APPLICATIONS OF ARTIFICIAL NEURAL NETWORKS IN ENGINEERING PROCESSING SYSTEM
}

S. Dadvandipour

Institute of Information Engineering, University of Miskolc, Egyetemváros, 3515, Miskolc, Hungary, e-mail: aitsamad@uni-miskolc.hu

\begin{abstract}
Artificial Neural Networks along with Image Processing Systems have proven to be successful, particularly in the domains of mathematics, science and technology. They have gained quite notable advantages beyond classical learning, as their usable engagement are observable in many fields of scientific environment related to the relevant systems. This paper presents a model for identifying the small components parts. The model may be significant in various industries mainly in engineering processing system areas. The objective of the study is to apply Artificial Neural Networks (ANN) in Image Processing System (IPS) with feed forward structure to detect, and recognize different parts or any other environment products on a moving conveyor bel. In the proposed model, we have used appropriate method of edge detection. The edge detection realizes artificial neural network with noise. The paper emphasizes the implementation of the model considering functionality, parts images, accurate detection and identifying the different components. The result shows that the model can detect moving objects (products of many kinds) accurately on the conveyor belt with very high success rate and sort them accordingly for further processes.
\end{abstract}

Keywords: process engineering, model, ANN, detection, products.

\section{INTRODUCTION}

Recognition is the classical problem in image processing, and machine vision. It is related to the determination of the image, which contains some specific objects, features, or activities. This operation can normally be solved robustly and without effort of a human, but is still not satisfactorily solved in machine vision for the general case, involving arbitrary objects in arbitrary situations. The existing methods for dealing with this task can solve it only for specific objects, such as simple geometric objects, human faces, printed or hand written characters. Furthermore in specific situations, typically described in terms of welldefined illumination, background, and pose of the object relative to the camera, $[1,2]$ and $[6,8]$. Artificial Neural Network (ANN), as a problem-solving tool, which imitates the process of human brain reactions has become an alternative method to recognize an image through training, [7]. MATLAB is the abbreviation of matrix laboratory, which has several hundred built-in functions packages and thirty kinds of tool kits. Many design, training and simulation functions of the Neural Network (NN) are provided in a NN toolbox. In this paper, we have used the MATLAB ANN Toolbox with Levenberg-Marquardt (LM) algorithm; and Feed Forward architecture. In ANN training process, the LM training function has less iteration than traditional Back Propagation (BP) and other improved algorithms while the convergence rate is faster and the precision is higher, [3, 4]. In feature extraction process, we extract five features for each object, $[6,7]$ and $[10]$ to recognize objects. Proposed method uses lower inputs to ANN and tends to higher efficiency of vision system. This method is suitable for real time recognition systems compared with previous research; because we can get better iteration time, speed of belt conveyor and accuracy. At this paper, we have also examined our search only on a small belt conveyor in order to see how fast the image processing happens with the proposed application of model, because our further work may be on material handling of the small parts using the same procedure.

\section{METHODOLOGY, HARDWARE AND SOFTWARE}

We have used a web-cam HD-6000 to capture the objects in conveyor belt. The captured image is processed by a program developed in C\#. NET environment. Feed forward neural network algorithms implemented by MATLAB functions, sending the output signals to the microcontroller ATMMEGA8. This microcontroller, which is connected to a personal computer via Universal Serial Bus, orders a servo motor's angle of operation to differentiate the small components in separate places. As regards the software development framework, the first level in the machine vision is the image processing algorithm, $[4,8]$. It would analyze and extract useful information from the image. The model of software structure is shown in Fig. 1 . 


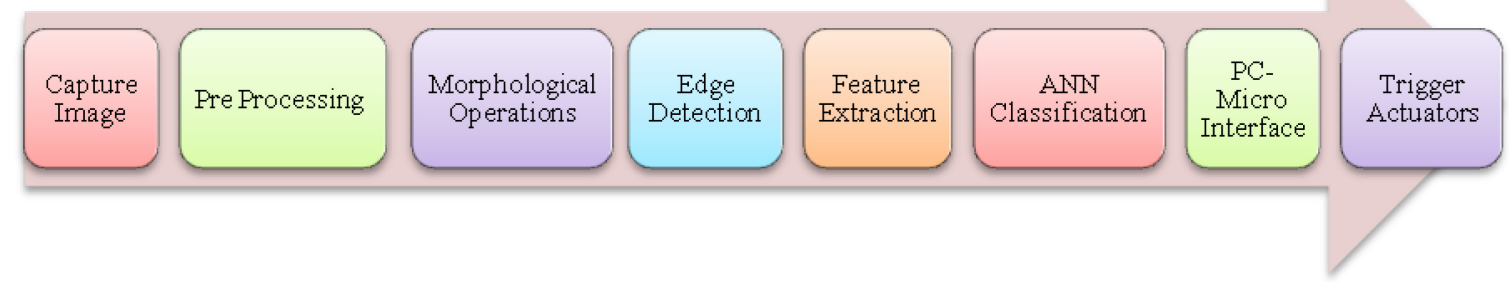

Figure 1. Software framework development structure

\subsection{Second-Level Heading}

Images captured by web-cam are in the RGB (Red, Green, and Blue) format, and because we do not need color information, the image is converted into grayscale color map. Using the adaptive threshold algorithm, the image is changed into binary form, which is suitable for further recognizing process, [2, 4] and [8].

\subsection{Image Pre-Processing}

The aim of the pre-processing is to make the blurred images become clear. Median filtering method can preserve edges and makes the noise out away, thus the image can be recovered well, [5, 9]. Median filtering, which can effectively suppress noise, is a nonlinear signal processing technology based on the sorting statistical theory. The method replaces the value of a pixel by the median of the gray levels in the neighborhood of that pixel. We usually use image sharpening treatment based on Laplace method in time domain. After pre-processing, the quality of image is improved significantly.

\subsection{Morphological Operations}

After pre-processing phase, in order to connect interrupted lines, we apply mathematical morphology dilation command. After this process, we clear the holes in image and eliminate noise coming from the outside line of the conveyor belt, $[5,9]$.

\subsubsection{Edge Detection and Feature Extraction}

Image which is processed by the first order differential equation usually produce relatively wide edge, so we use the gradient method based on the first derivative to enhance the edge of image. The approximate gradient of the image $\mathrm{f}(\mathrm{x}, \mathrm{y})$ is:

$$
\nabla^{\top} 2 f(x, y) \approx\left|G_{\perp} x\right|+\left|G_{\perp} y\right|=|\partial f / \partial x \|+| \partial f / \partial y \mid
$$

The above equation can be described by $3 \times 3$ filter mask shown in Fig. 2, and the approximate result is:

$\left.\left.\nabla f(x, y) \approx \mathbb{U}\left(a_{7}+2 a_{2}+a_{9}\right)-\left(a_{1}+2 a_{2}+a_{3}\right)|+|\left(a_{3}+2 a_{6}+a_{9}\right)-\left(a_{1}+2 a_{4}+a_{7}\right)\right]\right]$

In the above equation, we can find that the difference between the third row and first row is close to the differential in the x-direction, and the difference between the third column and first row is close to the differential in the y-direction, this can be expressed by $3 \times 3$ mask matrix illustrated in Fig. 3, where mask is called Sobel operator, [6-7]. After treatment by Sobel operator mask, the edge of image will be significantly more intuitive and the processed image is conducive to feature extraction. 


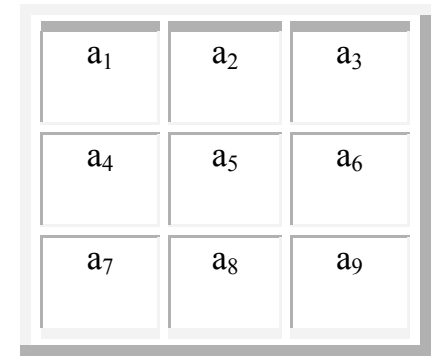

Figure 2. Filter mask structure $[3 \times 3]$
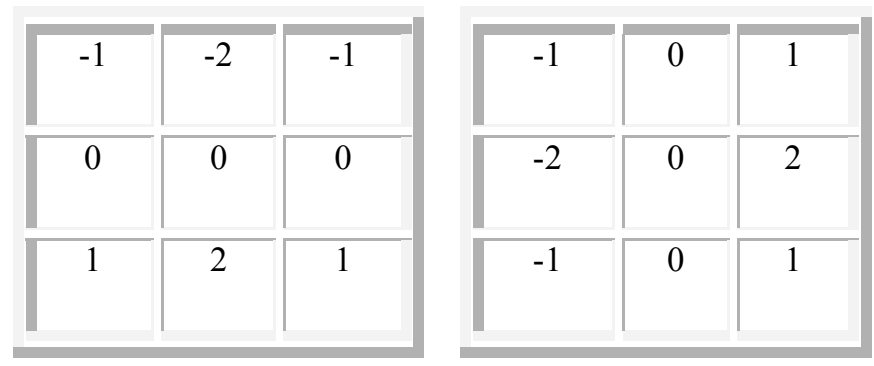

Figure 3. Sobel operator filter mask (left for vertical and right for horizontal edge detection)

Edge detection is done by using the Sobel operator to show the second shape of the object, [6-7]. After that we can extract two other features from object. If we calculate count of white pixels in image, we can define second feature as "PERIMETER", also by dividing "area" to "perimeter" we obtain third feature for objects. Then we define two other parameters as "length" and "width" by using length and width of detected object.

\subsubsection{Neural Network Architecture}

ANN has a multi- layers perceptron structure, which uses feed forward back propagation neural network. This structure has one input layer, one or more hidden layers and one output layer. The information enters to input layer and after manipulation is sent to output layers, $[9,3]$. The back-propagation algorithm uses the gradient of the performance function to determine how to adjust the weights to minimize errors that affect performance. In this paper, the activation function of each node uses a sigmoid function, $f(x)=1 /$ $(1+e-x)$. Sigmoid function generates values between $(0,1)$, so values are normalized before input the network and reduced between $(0,1)$. Feed forward training and application is shown in Fig. 4 . We define an input vector of size $1 * 5$ for neural network features generated in image processing phase. We tested some structures for our network by some parameters shown in Tab. 1. According to this table the obtained result achieved in method No. 9, by Mean Squared Error MSE = 2.0785e-12. Fig. 5 shows the training results obtained from Tab. 1 . 


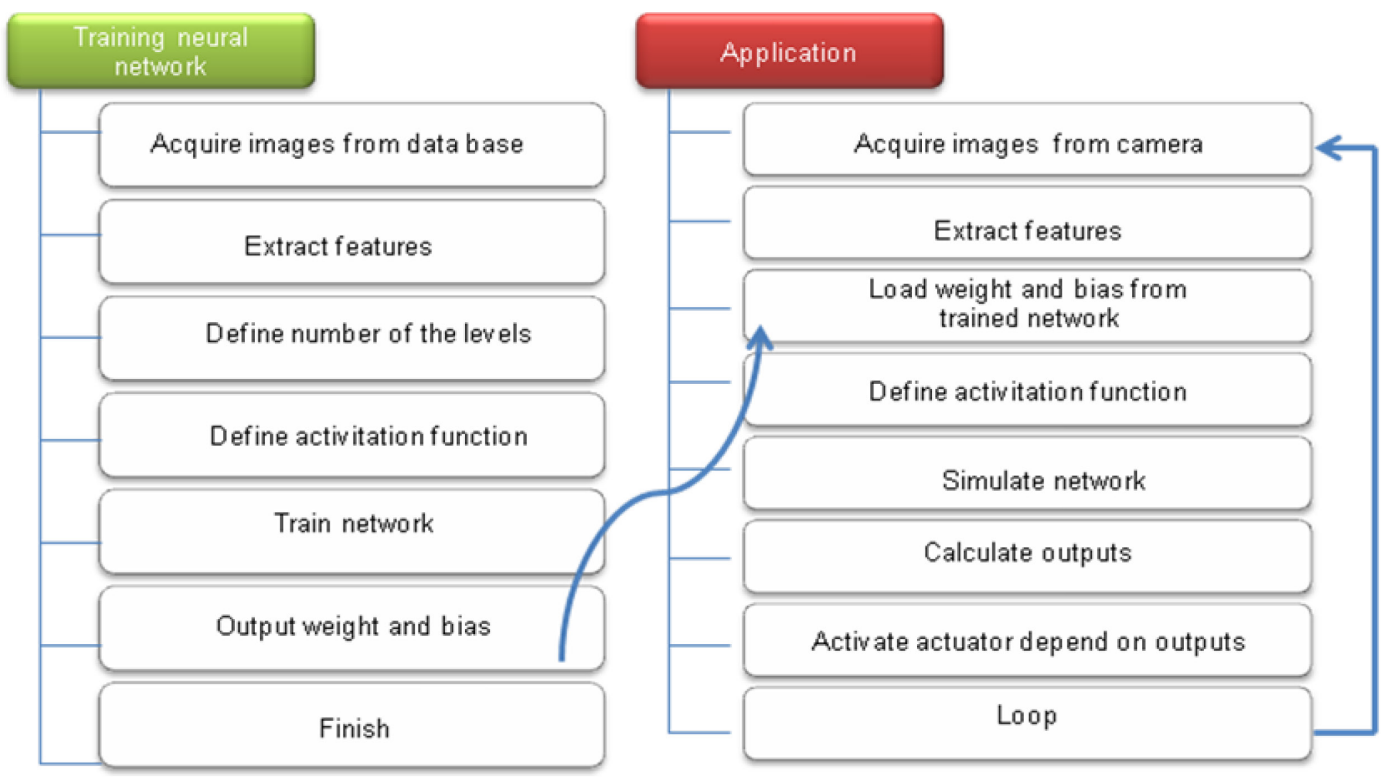

Figure 4.Training and application flowchart of Neural Network

Table 1. The heuristic results for different hidden layers and activation functions

\begin{tabular}{|cccccc|}
\hline Method & $\begin{array}{c}\text { Hidden } \\
\text { Layers }\end{array}$ & Neurons & Activation Functions & $\begin{array}{c}\text { Iterat } \\
\text { ion }\end{array}$ & MSE \\
\hline 1 & 1 & $5-3$ & tansig- purelin & 10 & 0.01118 \\
2 & 1 & $10-3$ & tansig- purelin & 15 & 0.01089 \\
3 & 1 & $15-3$ & tansig- purelin & 23 & 0.00378 \\
4 & 1 & $20-3$ & tansig- purelin & 16 & 0.01932 \\
5 & 1 & $25-3$ & tansig- purelin & 10 & $2.034 \mathrm{e}-5$ \\
6 & 1 & $30-3$ & tansig-purelin & 14 & 0.01009 \\
7 & 2 & $15-5-3$ & tansig-tansig-purelin & 20 & $3.79 \mathrm{e}-12$ \\
8 & 2 & $10-5-3$ & tansig-tansig-purelin & 14 & 0.00774 \\
$\mathbf{9}^{*}$ & $\mathbf{2}$ & $\mathbf{5 - 5}-3$ & tansig-tansig-purelin & $\mathbf{1 8}$ & $\mathbf{2 . 0 7 8 5 e - 1 2}$ \\
10 & 2 & $5-3-3$ & tansig-tansig-purelin & 21 & $1.765 \mathrm{e}-7$ \\
11 & 2 & $10-3-3$ & tansig-tansig-purelin & 50 & $3.295 \mathrm{e}-7$ \\
12 & 2 & $15-3-3$ & tansig-tansig-purelin & 15 & $2.977 \mathrm{e}-7$ \\
13 & 1 & $15-3$ & logsig-tansig & 13 & $7.73 \mathrm{e}-12$ \\
14 & 1 & $15-3$ & logsig-logsig & 18 & 0.00076 \\
15 & 1 & $15-3$ & purelin- logsig & 27 & $2.18 \mathrm{e}-10$ \\
\hline
\end{tabular}




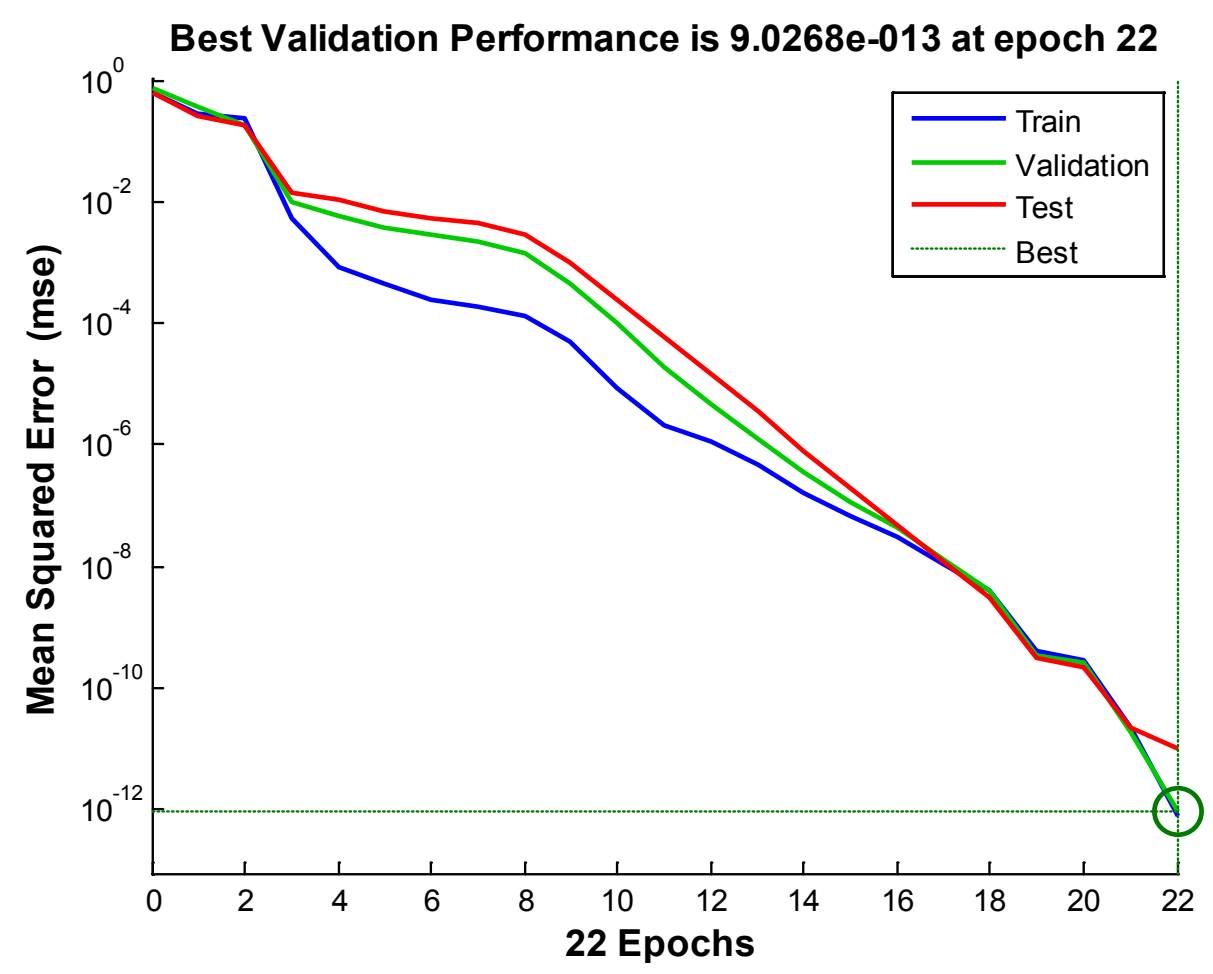

Figure 5. Output results of neural network training

\section{RESULT AND EVALUATION}

Several researchers have used image-processing techniques for object recognition. A series of morphological operations were implemented to produce only an image of nutmeat from an image containing a number of nuts. Color segmentation to locate and remove the long stems attached to mechanically harvested oranges. Their color segmentation algorithm had $100 \%$ success in discriminating the stemmed oranges. However, the algorithm misclassified some pixels of the stem-calyx as background. Some scientists used color machine vision for the detection of weeds in wheat and soybean fields. They used a color index for both the preprocessing and statistical analysis for weed detection. Their experiments worked well with statistical analysis compared to the two neural networks they trained. Rapid identification of objects with different features, materials and weight and color may be another important use of artificial intelligence and image processing system in recycling processing systems. This experimental work at this study shows satisfactory results as compared to the standard Artificial Neural Network technique while maintaining same threshold error and a good processing speed-up in terms of success rate, speed of conveyor belt, and types of machine tools. The success rate is quite good at 10 $\mathrm{cm} / \mathrm{second}$ for fixed speed of conveyor belt. This success rate with reference to literature used at this study, it is not a very bad result. But from author's point of view, a very satisfactory achievement might be performed in a place where there are no much fluorescent lighting, which of course increase the result ratio. The accepted and suitable results may obtain in an actual work place with its own conditions. In other word the correct and reasonable experimental test must be performed in an arbitrary place with arbitrary products of any kind. Then this paper might be evaluated to achieve the mentioned arbitrary materials with different colors, size, shape and features.

\section{CONCLUSION}

This paper focuses on the recognition system of small component parts like (machine tools) on a moving belt conveyor in real time. Mathematical analysis shows that edge detection is realized using artificial neural network (ANN) with noise. Supervised learning method with momentum is used. Laplacian edge detector is a teacher of artificial neural network. In this study, it is shown that Laplacian edge method can be used for training of ANN as edge detector. Testing is done using a real-time visual recognition system. 
The MATLAB software is used to integrate all algorithms (developed from image processing algorithms and ANN supervised learning method). Concerning the edge detection, some tools were used as extracted features.

\section{REFERENCES}

[1] R. Mattone et al, 2000, Sorting of Items on a Moving Conveyor Belt, Part 1: A technique for detecting and classifying objects. Robot Comput Integr Manuf, pp. 16:78-80.

[2] H. Isil Bozma et al, 2002, Visual Processing and Classification of Items on a Moving Conveyor, a selective perception approaches. Robot Comput Integr Manuf; 18(2), pp. 25-133.

[3] EN. Malamas et al, 2003, A Survey on Industrial Vision Systems, applications, pp. 21-88.

[4] H. Golnabi et al, 2007, Design and application of industrial machine vision systems, Robot Compute Integr Manuf, 23(6), pp. 630-7.

[5] H. Akbar et al, 2008, Image processing algorithm in machine vision approach for industrial inspection, in Proc. the 1st Makassar International Conference on Electrical Engineering and Informatics (MICEEI'08), pp. 58-62.

[6] A. Prabuwono et al, 2009, PC based weight scale system with load cell for product inspection, in Proc. International Conference on Computer Engineering and Technology (ICCET'09), pp. 343346.

[7] Z. Zhao et al, 2010, Application and Comparison of BP Neural Network Algorithm in MATLAB, in Proc. International Conference on Measuring Technology and Mechatronics Automation, pp. 590-593.

[8] I. Topalova et al, 2010, Increasing the Image Recognition Accuracy In Machine Vision Systems with Added Noise due to Technological Issues, IEEE 26-th Convention of Electrical and Electronics Engineers, pp. 328-332.

[9] T. Muhammad et al, 2011, Recognition of Bolt and Nut using Artificial Neural Network, International Conference on Pattern Analysis and Intelligent Robotics, pp. 165-1.

[10] Scavino et al. 2009, Application of automated image analysis to the identification and extraction of recyclable plastic bottles, Journal of Zhejiang University SCIENCE A, pp. 794-799. www.springerlink.com. 